\title{
Dosimetric Comparison between Three Dimensional Conformal Radiation Therapy (3DCRT) \& Intensity Modulated Radiation Therapy (IMRT) in Mid-Lower Oesophageal Carcinoma
}

\author{
Azza N. Taher'1, Rasha A. Elawady², Amr Amin'1 \\ ${ }^{1}$ The Department of Radiation Oncology, National Cancer Institute, Cairo University, Cairo, Egypt \\ ${ }^{2}$ The Unit of Radiation Physics, Department of Radiation Oncology, National Cancer Institute, Cairo University, Cairo, Egypt \\ Email: ataher3@yahoo.com
}

How to cite this paper: Taher, A.N., Elawady, R.A. and Amin, A. (2019) Dosimetric Comparison between Three Dimensional Conformal Radiation Therapy (3DCRT) \& Intensity Modulated Radiation Therapy (IMRT) in Mid-Lower Oesophageal Carcinoma. International Journal of Medical Physics, Clinical Engineering and Radiation Oncology, 8, 121-129.

https://doi.org/10.4236/ijmpcero.2019.82011

Received: February 19, 2019

Accepted: May 24, 2019

Published: May 27, 2019

Copyright $\odot 2019$ by author(s) and Scientific Research Publishing Inc. This work is licensed under the Creative Commons Attribution International License (CC BY 4.0).

http://creativecommons.org/licenses/by/4.0/

\section{Open Access}

\begin{abstract}
Purpose: To investigate if intensity modulated radiation therapy (IMRT) offers a better planning target volume (PTV) coverage and/or lower dose to normal thoracic structures in comparison to three dimensional conformal radiation therapy (3DCRT) in the treatment of mid and lower oesophageal carcinoma patients. Materials and Methods: A prospective study in the period from 2014 till 2015 was held in the radiation therapy department of the National Cancer Institute, Cairo University, in which 20 locally advanced or inoperable mid and lower oesophageal cancer patients were treated by chemo-radiation using 3DCRT technique. IMRT plans were generated for those 20 patients. The 3DCRT and IMRT plans were compared as regards PTV coverage and doses to critical organs at risk. Results: All plans had produced satisfactory PTV coverage with no significant differences noted. The lung V20 for both lungs in 3DCRT was $16.94 \% \pm 4.2 \%$ which was increased to $21.42 \% \pm 3.6 \%$ in IMRT $(\mathrm{p}=0.017)$. The mean dose to the heart and V30 were higher in IMRT plans while the mean dose to the spinal cord was higher with 3DCRT plans, yet that didn't reach a statistically significant level ( $\mathrm{p}=$ 0.156). The dose delivered to the liver didn't pose any difference between both techniques. Conclusion: 3DCRT remains to be a feasible cost effective treatment delivery option for mid and lower oesophageal cancer cases with a lower optimization and delivery time than that for IMRT. Moreover, that calls for further dosimetric studies and clinical trials to assess IMRT technique. In our study, IMRT using nine fields didn't prove to be superior to 3DCRT.
\end{abstract}




\section{Keywords}

Oesophageal Carcinoma, 3DCRT, IMRT, Dosimetric

\section{Introduction}

Oesophageal cancer continues to rank as one of the highly aggressive and lethal gastrointestinal diseases globally [1]. Poor treatment outcomes continue to challenge the multidisciplinary array of surgeons, medical and radiation oncologists.

Most patients are present in an advanced or an unresectable stage [2]. This fact has led to the establishment of concurrent chemo radiotherapy (CCRT) as the staple treatment policy for such cases [3]. Moreover, local failure remains to be the commonest failure pattern coupled by local persistence of the tumor [4].

The technique of radiation therapy delivery has evolved along the years starting with the basic antero-posterior/postero-anterior (AP/PA) field arrangement, then the 4 fields box technique reducing the lateral fields' weight to decrease dose to lungs, as well as the 3-field technique; anteroposterior field and 2 posterior oblique fields. Up until the era of the $3 \mathrm{D}$ conformal radiation therapy (3DCRT) has become the technique of choice for many years now in various centers [5].

Since locoregional failure or persistence after treatment reaches 50\% [4] [6] that raised the flag for delivering higher radiation dose to increase local control. Meanwhile, critical surrounding normal tissue tolerance must be respected, namingly the lungs, spinal cord and heart specially that the planning target volume (PTV) is central. Various studies reported good dosimetry and patient outcome by IMRT [7] [8]. The main disadvantages of IMRT despite its being effective in dose conformity to tumor are increased treatment delivery time and monitor units (MU).

We conducted this prospective study in our department to compare the dose distribution for the PTV and organs at risk (OAR) using the 3DCRT and those were compared with the IMRT generated plans.

\section{Materials and Methods}

\section{Patients Data and Simulation}

This is a prospective dosimetric study conducted in the radiation therapy department of the National Cancer Institute, Cairo University, Egypt. 20 patients with locally advanced histopathologically proven mid and lower oesophageal carcinoma, not reaching gastro-esophageal junction were treated with chemoradiation from the period of April 2014 till July 2015.

Patients were aged from 48 to 70 years, they all had histopathologically proven oesophageal Squamous cell carcinoma.

Patients were simulated with a General Electric Lightspeed RT 16 computed tomography simulator with $2.5 \mathrm{~mm}$ slices. Patients were asked to lie in a supine 
position with both arms raised over their heads.

Treatment planning was done on XIO (CMS) treatment planning system version 5.1

A gross tumor volume (GTV) covering the gross oesophageal tumor and positive regional lymph nodes was contoured. The clinical target volume (CTV) encompassed a proximal and distal margin of $5 \mathrm{~cm}$ and a radial margin of $15 \mathrm{~mm}$ added to the GTV. The planning target volume (PTV) varied from case to case yet usually averaged $10 \mathrm{~mm}$ all around the CTV to account for organ movement. OAR included the heart, lungs, spinal cord and liver. Figure 1 shows dose distribution for one of our mid oesophageal cancer cases for both the 3DCRT and IMRT plans.

3DCRT plan were created using XIO treatment planning system $6 \mathrm{MV}$ photons. 3 - 5 fields were shaped at the beam's eye view to encompass the PTV shape using MLC at gantry angles of $0^{\circ}, 90^{\circ}, 270^{\circ}$ or $0^{\circ}, 45^{\circ}, 90^{\circ}, 270^{\circ}$ and $315^{\circ}$ The treatment target volume included PTV and an additional $0.7 \mathrm{~cm}$ margin for beam penumbra in all directions. Physical and virtual wedges were used to modify the dose in the treatment plan and to perform dose homogeneity in PTV. The prescribed dose was $1.8 \mathrm{~Gy} \times 28$ fractions for a total dose of $50.4 \mathrm{~Gy}$.

IMRT plan was performed using Monaco treatment planning system $6 \mathrm{MV}$ photons with 9 equally spaced coplanar beams using commercial inverse planning software and avoid opposing fields. For inverse-planned 9F-IMRT, the gantry angles were $0^{\circ}, 40^{\circ}, 80^{\circ}, 120^{\circ}, 160^{\circ}, 200^{\circ}, 240^{\circ}, 280^{\circ}$ and $320^{\circ}$.

The organs at risk dose constraints given for lungs, heart, spinal cord and liver for planning and optimization are shown in Table 1 [9].

A 9 beam IMRT plan was generated for all patients considering same contouring. All plans aimed to achieve a min. dose $>95 \%$ and $\max$. dose $<107 \%$

Statistical analysis was performed using SPSS version 22.0 software (Chicago, IL, USA) and SAS version 9.4. Significance level was set at $\mathrm{p} \leq 0.05$ (Figure 1).

\section{Results}

The 3DCRT and IMRT plans were dosimetrically evaluated, dose coverage to PTVs all techniques achieved the constraint that $95 \%$ of the volume is covered by more than $95 \%$ of the prescribed dose. Dose homogeneity within the various PTVs was comparable with no statistically significant difference between both techniques (Table 2).

As for the organs at risk (OAR) the mean dose to the heart and the V30 were both higher in the IMRT plans without reflecting any statistically significant difference and not exceeding the dose constraints where the mean dose for 3DCRT was 12.6 Gy vs. 13.9 Gy for IMRT ( $\mathrm{p}=0.324$ ), and the Heart V30 was $14.5 \mathrm{~Gy}$ for 3DCRT vs. 19.6 Gy for IMRT technique plans ( $\mathrm{p}=0.116)$.

Despite the fact that the mean dose to the spinal cord delivered by IMRT was 34.5 Gy vs. 37.5 Gy with 3DCRT yet that didn't amount to a statistically significant difference $(\mathrm{p}=0.156)$. 
Table 1. Dose constraints for organs at Risk (OAR).

\begin{tabular}{cc}
\hline Structure & Constraint \\
\hline Lungs & V20 $\leq 35 \%$ \\
Heart & Mean dose $<26$ Gy \\
V $30<46 \%$ \\
Dmax $<45$ Gy \\
Spinal Cord & 45 Gy to whole SC with not more than $2 \%$ of SC $>50$ Gy \\
Whole Liver & Mean dose $<32-30$ Gy \\
\hline
\end{tabular}

Table 2. Average Dose-Volume Statistics for PTV for Both 3DCRT and IMRT Techniques.

\begin{tabular}{cccc}
\hline & 3DCRT (Gy) & IMRT (Gy) & p-Value \\
\hline Min. Dose & 40.7 & 39.8 & 0.47 \\
Max Dose & 52.8 & 56.2 & 0.06 \\
Mean Dose & 49.9 & 51.0 & 0.182 \\
\hline
\end{tabular}
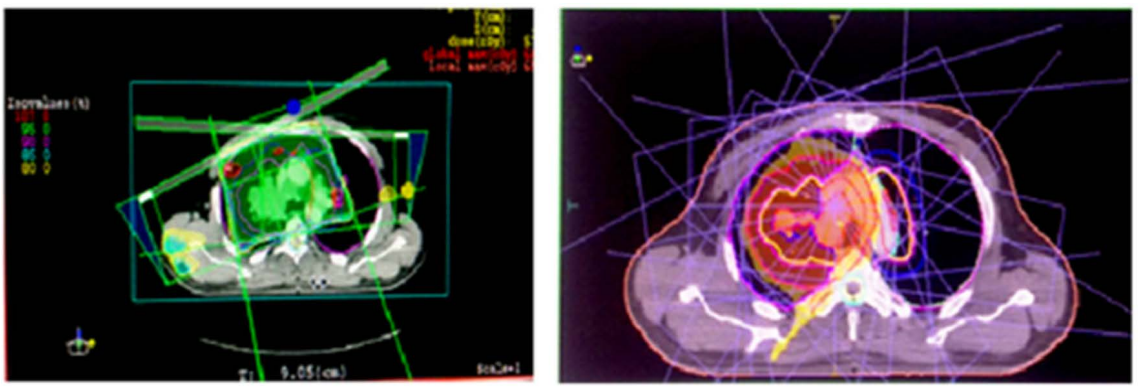

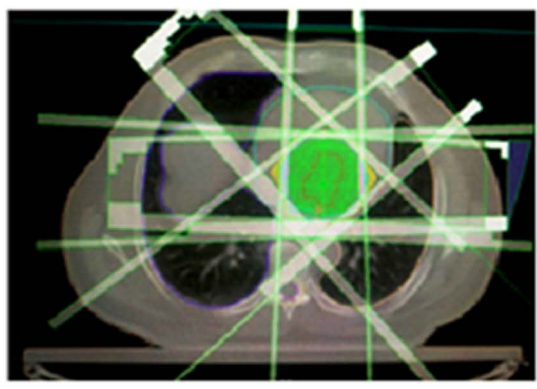

3DCRT

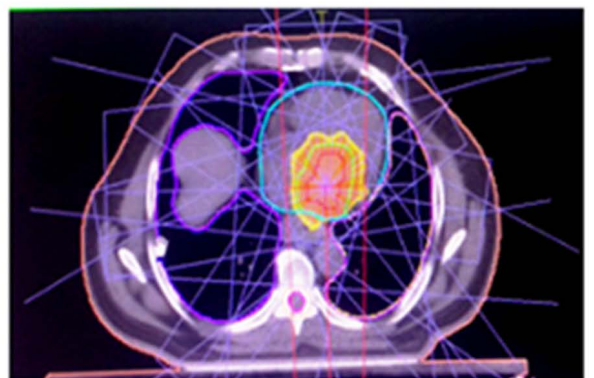

IMRI

Figure 1. Dose distributions of 3DCRT plan were created using XIO treatment planning system $6 \mathrm{MV}$ photons. 3 - 5 fields were shaped at the beam's eye view to encompass the PTV shape using MLC (left) and IMRT plan was performed using Monaco treatment planning system $6 \mathrm{MV}$ photons with 9 equally spaced coplanar beams (right) for a middle third oesophageal cancer in axial view.

The V20 for the 3DCRT plans delivered lesser lung volume irradiation; 16.9\% when compared to IMRT plans; $21.4 \%$ with a p-value of 0.017 denoting a statistically significant value in favor of the 3DCRT plans.

The mean dose delivered to the liver was $4.3 \mathrm{~Gy}$ for 3DCRT vs. $5.1 \mathrm{~Gy}$ for IMRT plans and that did not show any statistically significant difference between 
3DCRT and IMRT arms $(\mathrm{p}=0.192)$.

\section{Discussion}

In order to tackle the pitfall of low survival rates and after various randomized trials, concomitant chemoradiation has become a standard treatment in oesophageal cancer patients producing up to $25 \%-30 \% 5$ year survival rates [10] [11]. Yet again studies showed that chemoradiation can come with a high price tag of severe complications [4] [6].

Thus we designed current study to address the question of dosimetric differences between IMRT and 3DCRT for mid and lower esophageal cancers, and to evaluate if IMRT can be implemented for dose escalation to the target volume without increasing the dose to various organs at risk specially the lungs.

Current study didn't show any significant improvement in the PTV coverage by IMRT compared to 3DCRT and this finding matches the results from the study published by Ghosh et al. [12] where the dose homogeneity was comparable for both 3DCRT and IMRT techniques. Similarly the study by Wu et al. [13] reported no significant superiority of IMRT or even Volumetric Modulated Arc Therapy (VMAT) plans over 3DCRT in middle oesophageal cancer cases. Our findings differ from the results of the study by Fenkell et al. [5]; where they compared IMRT with 3DCRT in the treatment of the cervical esophageal cancer, the median coverage of various PTVs even 50 and 70 were all improved with IMRT. This discrepancy could be explained by the relatively simpler shape of the target for our middle and lower esophageal cancers versus that of cancers located in the cervical oesophagus in Fenkell's study. On the other hand Nutting et al. [14] concluded that the dose conformity of IMRT and VMAT was improved for middle esophageal cancer when compared to 3DCRT. The study of Vivekanandan et al. [15] again showed superiority of IMRT and VMAT in target dose conformity versus 3DCRT in esophageal cancer. Though they didn't specify which segment of the oesophagus did they study.

As early as 1999, Khoo et al. [16] reported that the superiority of IMRT with its dose painting ability was more established for complex targets; as with the more complicated target coverage of head and neck cancers compared to 3DCRT, in oesophageal cases the greatest benefit was seen when the tumor was concave, thus in most cases of oesophageal carcinoma cases where the PTV is mostly cylindrical that minimizes the benefit from IMRT. The dose deliver for patient in 3DCRT technique is easier than IMRT. It is also lower in cost and less time-consuming compared with IMRT [17] [18].

The results of this study match these algorithms where there was a small benefit as regards sparing of spinal cord yet none seen as regards the heart or lungs; where IMRT shows superior conformality to treatment volume thus delivering higher doses yet at the expense of increased number of beams delivering small radiation doses to greater normal tissue volume [5]. This fact had a clear impact on our results since our patients had advanced oesophageal tumors producing a 
large PTV alongside large volume of surrounding organs at risk specially lungs and spinal cord. We generated 9 beam IMRT plans and compared them to the original 3DCRT treatment plans, in our case the 9 beams were equispaced as in the study by Nutting et al. [19], who concluded no benefit in dose reduction to the lungs in their IMRT plans, still we shared same conclusion and our lungs V20 was statistically significantly higher with the IMRT plans. Moreover, Ghosh et al. [12] reported statistically significant higher lung V20 with IMRT which is in accordance with our results. As for Chandra et al. [7] they compared 4, $7 \& 9$ IMRT beem plans to 3DCRT in lower oesophageal cancer patients and they reported a 5\% reduction in lung V20 with IMRT plans which is different than our findings. Similarly, Wu et al. [13] reported a lower lung V20 with IMRT yet not reaching a statistically significant value. Nutting et al. [19] reported a reduction in mean lung dose upon using a 4 field IMRT when compared to the 9 fields IMRT and the 3DCRT plans.

On the other hand, in current study IMRT delivered lower mean dose to the spinal cord but not reaching a statistically significant level, this was also reported by Ghosh et al. [12] and also consistent with the results reported by Vivekeanandan et al. [15].

Current study results showed lower mean dose to the heart with 3DCRT when compared with IMRT and the V30 was also lower with 3DCRT still neither of those values reached a statistically significant value. Similar results were published by Candra et al. [7]. Chen et al. [20] also published a study on a dosimetric analysis of 10 mid-distal esophageal carcinoma cases comparing helical tomotherapy, step-and-shoot IMRT and 3DCRT, the IMRT plans resulted in decreased heart V30 and V45. Our findings are also consistent with Wu et al. [13] regarding the V30 heart sparing effect with 3DCRT that reached a statistically significant value. Mayo et al. [21] supported the same findings as well. The study by Ghosh et al. [12] stands to differ with our findings as they reported higher mean heart dose with 3DCRT yet not exceeding the dose constraints.

Another organ at risk is the liver that surrounds part of the oeosophageal circumference distally thus the more coplanar beams implemented the more the dose that will reach the liver as our results showed higher mean dose delivered with the IMRT plans (Table 3). Our results are consistent with the findings published by Chandra et al. [7]. Also Ghosh et al. [12] reported that IMRT delivered a higher mean dose to the liver than with the 3DCRT in their lower oesophageal cancer cases though not of statistical significance same as our cases.

\section{Conclusion}

3DCRT can be reliably applied in the treatment of in Mid-Lower Oesophageal carcinoma, as it is easy to deliver, low in cost and time-saving compared to IMRT. It provides homogenous doses to the target and good sparing of OARs. IMRT did not produce any dosimetric advantage over the 3DCRT technique, apart from the decreased mean dose to the spinal cord. On the contrary, IMRT 
Table 3. Dose statistics extracted from the DVHs of Organs at Risk (OAR) for 3DCRT and IMRT techniques.

\begin{tabular}{cccc}
\hline & 3DCRT & IMRT & p-value \\
\hline Heart mean dose V30 & $12.6 \mathrm{~Gy}$ & $13.9 \mathrm{~Gy}$ & 0.324 \\
Spinal Cord & $14.5 \%$ & $19.6 \%$ & 0.116 \\
Both lungs V20 & $37.5 \mathrm{~Gy}$ & $34.5 \mathrm{~Gy}$ & 0.156 \\
Liver & $16.9 \%$ & $21.4 \%$ & 0.017 \\
\hline
\end{tabular}

technique poses a higher chance of lung toxicity compared to 3DCRT which is so far a better choice in combined chemo-radiation therapy in oesophageal cancer cases by virtue of decreasing lung dose as well as being cost effective in a busy radiation therapy department. In order to consider implementing IMRT with or without escalated dose for the treatment of oesophageal cancer further clinical trials and dosimetric studies are called for.

\section{Conflicts of Interest}

The authors declare no conflicts of interest regarding the publication of this paper.

\section{References}

[1] Jemal, A., Siegel, R. and Ward, E. (2008) Cancer Statistic. CA: A Cancer Journal for Clinicians, 58, 71-96. https://doi.org/10.3322/CA.2007.0010

[2] Kaifi, J.T., Gusani, N.J. and Jiang, Y. (2011) Multidisciplinary Management of Early and Locally Advanced Esophageal Cancer. Journal of Clinical Gastroenterology, 45, 391-399. https://doi.org/10.1097/MCG.0b013e3182049949

[3] Tepper, J., Krasna, M.J. and Niedzwiecki, D. (2008) Phase III Trial of Trimodality Therapy with Cisplatin, Fluorouracil, Radiotherapy, and Surgery Compared with Surgery Alone for Esophageal Cancer: CALGB 9781. Journal of Clinical Oncology, 26, 1086-1092.

[4] Cooper, J., Guo, M., Herskovic, A., Macdonald, J.S., Martenson, J.A. and Al-Sarraf, M. (1999) Chemoradiotherapy of Locally Advanced Esophageal Cancer: Long-Term Follow-Up of a Prospective Randomized Trial (RTOG 85-01). The Journal of the American Medical Association, 281, 1623-1627. https://doi.org/10.1001/jama.281.17.1623

[5] Fenkell, L., Kaminsky, I., Breen, S., Huang, S., Van Prooijen, M. and Ringash, J. (2008) Dosimetric Comparison of IMRT vs. 3D Conformal Radiotherapy in the Treatment of Cancer of the Cervical Esophagus. Radiotherapy and Oncology, 89, 287-291. https://doi.org/10.1016/j.radonc.2008.08.008

[6] Minsky, B., Pajak, T., Ginsberg, R., Pisansky, T.M., Martenson, J. and Komaki, R. (2002) INT 0123 (Radiation Therapy Oncology Group 94-05) Phase III Trial of Combined Modality Therapy for Esophageal Cancer: High-Dose versus Standard-Dose Radiation Therapy. Journal of Clinical Oncology, 20, 1167-1174. https://doi.org/10.1200/JCO.2002.20.5.1167

[7] Chandra, A., Guerrero, T.M., Liu, H.H., Tucker, S.L., Liao, Z. and Wang, X. (2005) 
Feasibility of Using Intensity-Modulated Radiotherapy to Improve Lung Sparing in Treatment Planning for Distal Esophageal Cancer. Radiotherapy and Oncology, 77, 247-253. https://doi.org/10.1016/j.radonc.2005.10.017

[8] Staffurth, J. and Radiotherapy Development Board (2010) A Review of the Clinical Evidence for Intensity-Modulated Radiotherapy. Clinical Oncology, 22, 643-657.

[9] Marks, L.B., Yorke, E.D. and Jackson, A. (2010) Use of Normal Tissue Complication Probability Models in the Clinic. International Journal of Radiation Oncology, Biology, Physics, 76, S10-S19. https://doi.org/10.1016/j.ijrobp.2009.07.1754

[10] Bosset, J., Gignoux, M., Triboulet, J., Tiret, E., Mantion, G. and Elias, D. (1997) Chemoradiotherapy Followed by Surgery Compared with Surgery Alone in Squamous Cell Cancer of the Esophagus. The New England Journal of Medicine, 337, 161-167. https://doi.org/10.1056/NEJM199707173370304

[11] Al-Sarraf, M., Martz, K., Herskovic, A., Leichman, L., Brindle, J.S., Vaitkevicius, V.K., et al. (1997) Progress Report of Combined Chemoradiotherapy versus Radiotherapy Alone in Patients with Esophageal Cancer: An Intergroup Study. Journal of Clinical Oncology, 15, 277-284. https://doi.org/10.1200/JCO.1997.15.1.277

[12] Ghosh, S., Kapoor, R., Gupta, R., Khosla, D., Kochhar, R., Oinam, A.S., Sharma, R. and Sharma, S.C. (2012) An Evaluation of Three Dimensional Conformal Radiation Therapy versus Intensity Modulated Radiation Therapy in Radical Chemoradiation of Esophageal Cancer: A Dosimetric Study. Clinical Cancer Investigation Journal, 1, 65-70. https://doi.org/10.4103/2278-0513.99565

[13] Wu, Z., Xie, C., Hu, M., Han, C. and Yi, J. (2014) Dosimetric Benefits of IMRT and VMAT in the Treatment of Middle Thoracic Esophageal Cancer: Is the Conformal Radiotherapy Still an Alternative Option? Journal of Applied Clinical Medical Physics, 15, 93-101.

[14] Nutting, C.M., Dearnalay, D.P. and Webb, S. (2000) Intensity Modulated Radiation Therapy: A Clinical Review. The British Journal of Radiology, 73, 459-469. https://doi.org/10.1259/bjr.73.869.10884741

[15] Vivekanandan, N., Sriram, P., Kumar, S., Bhuvaneswari, N. and Saranya, K. (2012) Volumetric Modulated Arc Radiotherapy for Esophageal Cancer. Medical Dosimetry, 37, 108-113. https://doi.org/10.1016/j.meddos.2011.01.008

[16] Khoo, V.S., Oldham, M., Adams, E.J., Bedford, J.L., Webb, S. and Brada, M. (1999) Comparison of Intensity-Modulated Tomotherapy with Stereotactically Guided Conformal Radiotherapy for Brain Tumors. International Journal of Radiation Oncology, Biology, Physics, 45, 415-425. https://doi.org/10.1016/S0360-3016(99)00213-8

[17] Cheung, K.Y. (2006) Intensity Modulated Radiotherapy: Advantages, Limitations and Future Developments. Biomedical Imaging and Intervention Journal, 2, e19. https://doi.org/10.2349/biij.2.1.e19

[18] Yong, J.H.E., McGowan, T., Redmond-Misner, R., et al. (2016) Estimating the Costs of Intensity-Modulated and 3-Dimensional Conformal Radiotherapy in Ontario. Cancer Research, 23, 228-238. https://doi.org/10.3747/co.23.2998

[19] Nutting, C.M., Bedford, J.L., Cosgrove, V.P., Tait, D.M., Dearnaley, D.P. and Webb, S. (2001) A Comparison of Conformal and Intensity-Modulated Techniques for Oesophageal Radiotherapy. Radiotherapy and Oncology, 61, 157-163. https://doi.org/10.1016/S0167-8140(01)00438-8

[20] Chen, Y.J., Liu, A. and Han, C. (2007) Helical Tomotherapy for Radiotherapy in Esophageal Cancer: A Preferred Plan with Better Conformal Target Coverage and More Homogeneous Dose Distribution. Medical Dosimetry, 32, 166-171. 
https://doi.org/10.1016/j.meddos.2006.12.003

[21] Mayo, C.S., Urie, M.M., Fitzgerald, T.J., Ding, L., Lo, Y.C. and Bogdanov, M. (2008) Hybrid IMRT for Treatment of Cancers of the Lung and Esophagus. International Journal of Radiation Oncology, Biology, Physics, 71, 1408-1418.

https://doi.org/10.1016/j.ijrobp.2007.12.008 